\title{
A Methodology for Sharing Archival Descriptive Metadata in a Distributed Environment
}

\author{
Nicola Ferro and Gianmaria Silvello \\ Department of Information Engineering, University of Padua, Italy \\ \{ferro, silvello\}@dei.unipd.it
}

\begin{abstract}
This paper discusses how to exploit widely accepted solutions for interoperation, such as the pair Open Archives Initiative Protocol for Metadata Harvesting (OAI-PMH) and Dublin Core (DC) metadata format, in order to deal with the peculiar features of archival description metadata and allow their sharing. We present a methodology for mapping Encoded Archival Description (EAD) metadata into Dublin Core (DC) metadata records without losing information. The methodology exploits Digital Library System (DLS) technologies enhancing archival metadata sharing possibilities and at the same time considers archival needs; furthermore, it permits to open valuable information resources held by archives to the wider context of the cross-domain interoperation among different cultural heritage institutions.
\end{abstract}

\section{Introduction}

The role of DLSs in collecting, managing and preserving our cultural heritage is increasingly preponderant in several contexts. In particular, DLSs are not merely the digital counterpart of traditional libraries, rather they can be seen as the technology of choice for managing the information resources of different kinds of organizations, which range from libraries, and museums to archives. In this wider context, the need for interoperability among different systems is becoming a compelling issue which is being faced by several initiatives.

The DELOS Network of Excellence on Digital Libraries 1 has proposed and developed a reference model for laying the foundations of digital libraries 3 which takes into account the perspectives and needs of different cultural heritage institutions and provides a coherent view on the main concepts which constitute the universe of digital libraries in order to facilitate the co-operation among different systems. Furthermore, the Open Archives Initiative (OAI) promotes interoperability through OAI-PMH [22, a flexible and lightweight protocol for metadata harvesting, which is becoming the de-facto standard in metadata exchange in distributed environments. This protocol permits metadata harvesting between different repositories in a straightforward fashion, in order to create aggregated metadata collections and to enable the creation of advanced services on them. The Digital Repository Infrastructure Vision for European Research (DRIVER)

\footnotetext{
1 http://www.delos.info/

B. Christensen-Dalsgaard et al. (Eds.): ECDL 2008, LNCS 5173, pp. 268 2792008.

(C) Springer-Verlag Berlin Heidelberg 2008
} 
project2 levers also on the OAI-PMH protocol in order to develop a pan-European Digital Repository Infrastructure by integrating existing individual repositories from European countries and developing a core number of services, including search, data collection, profiling and recommendation [4.

Finally, the "European Commission Working Group on Digital Library Interoperability", active from January to June 2007, had the objective of providing recommendations for both a short term and a long term strategy towards "the setting up of the European Digital Library as a common multilingual access point to Europe's distributed digital cultural heritage including all types of cultural heritage institutions" 8 . In particular, the recipient of this recommendations is the EDLnet thematic network 3 , which aims at addressing the interoperability issues among European museums, archives, audio-visual archives and libraries towards the creation of the "European Digital Library".

In this paper, we have been carrying on our work on the design and development of a distributed DLS for sharing archival description metadata [1/2] and we discuss how to exploit widely accepted solutions for interoperation, such as the pair OAI-PMH and $\mathrm{DC}^{4}$ metadata format, in order to deal with the peculiar features of archival description metadata and allow their sharing. In particular, we describe how archival descriptions encoded using the EAD [13], a commonly accepted metadata format in the archival domain, can be successfully translated into DC records to be shared and harvested through OAI-PMH without losing any information of the original EAD encoding and instead gaining greater flexibility. The proposed solution can be useful not only for improving the cooperation among archival systems themselves but also for opening the valuable information resource held by archives to the wider context of the cross-domain interoperation among different cultural heritage institutions.

The paper is organized as follows: Section 2 introduces some background information about archival description metadata and some requirements they have to meet in order for them to be effectively shared. Section 3 describes the features of the EAD metadata format that are relevant to our proposal; Section 4 introduces both the OAI-PMH protocol and the DC metadata format and highlights what of their characteristics will be levered in our propostal; Section 5 reports our proposed methodology for managing archival description metadata in a distributed environment; finally, Section 6 draws some conclusions.

\section{Archives and Archival Descriptions}

An archive is not simply constituted by a series of objects that have been accumulated and filed with the passing of time but, on the contrary, it represents the trace of the activities of a physical or juridical person in the course of their business which is preserved because of their continued value. Archives have to

2 http://www.driver-repository.eu/

3 http://www . europeandigitallibrary.eu/edlnet/

4 http://www.dublincore.org/ 
keep the context in which their record 5 have been created and the network of relationships among them in order to preserve their informative content and provide understandable and useful information over time.

Archival description is defined in [14] as "the process analyzing, organizing, and recording details about the formal elements of a record or collection of records, to facilitate the work's identification, management, and understanding"; archival descriptions have to reflect the peculiarities of the archive, retain all the informative power of a record, and keep trace of the provenance and original order in which resources have been collected and filed by archival institutions [7. This is emphasized by the central concept of fonds, which should be viewed primarily as an "intellectual construct", the conceptual "whole" that reflects an organic process in which a records creator produces or accumulates series of records [5. In this context, provenance becomes a fundamental principle of archives; the principle of the "respect des fonds" which dictates that resources of different origins be kept separate to preserve their context.

6] highlights that maintaining the provenance leads archivists to evaluate records on the basis of the importance of the creator's mandate and functions, and fosters the use of a hierarchical method. The hierarchical structure of the archive expresses the relationships and dependency links among the records of the archive by using what is called the archival bond6. Archival bonds, and thus relations, are constitutive parts of an archival record: if a record is taken out from its context and lost its relations, its informative power would also be considerably affected. Therefore, archival descriptions need to be able to express and maintain such structure and relationships in order to preserve the context of a record. To this end, the International Council on Archives (ICA) 7 has developed a general standard for archival description called International Standard for Archival Description (General) (ISAD $(G))$ 9]. According to $\operatorname{ISAD}(\mathrm{G})$, archival description proceeds from general to specific as a consequence of the provenance principle and has to show, for every unit of description, its relationships and links with other units and to the general fonds. Therefore, archival descriptions produced according to the $\operatorname{ISAD}(G)$ standard take the form of a tree which represents the relationships among more general and more specific archive units going from the root to the leaves of the tree.

Archives may benefit greatly from the digitalization of their resources. One of the most significant improvements is the possibility of sharing archival resources between several repositories in different geographical locations and create value-added services on the shared resources, in order to provide broad and easy access to them. What can be usually shared in digital and distributed environments are the archival descriptions, which are encoded according to a metadata schema. The use of metadata allows us to exploit DLS technologies and data

\footnotetext{
${ }^{5}$ In [12] a record is defined as: "Any document made or received and set aside in the course of a practical activity".

${ }^{6}$ In 14 the archival bond is defined as: "The interrelationships between a record and other records resulting from the same activity."

7 http://www.ica.org/
} 
exchange protocols and apply them to the archival descriptions. In order to respect ISAD $(\mathrm{G})$ principles and to support DLS advanced services, archival description metadata should meet the following three main requisites:

1. Context: archival description metadata have to retain information about the context of a given record, such as the relations between records and with the production environment, as stated by the respect des fonds principle discussed above.

2. Hierarchy: archival description metadata have to reflect the archive organization which is described in a multi-leveled fashion, as defined by $\operatorname{ISAD}(G)$.

3. Variable granularity: archival description metadata have to facilitate access to the requested items, which may belong to different hierarchical levels, with the desired degree of detail and without requiring access to the whole hierarchy.

Moreover, we have to take into account one of the main objectives that is metadata sharing; in order to enable archival metadata to be shared by means of a protocol for metadata exchange, they have also to respect protocol needs regarding metadata format and size. We have to take into account both the necessities of archival descriptions expressed by a certain metadata format and the constraints of the protocol chosen for sharing the metadata. These two main aspects need to be weighed up in order to exploit the possibilities offered by a digital and distributed environment and to satisfy all archival information needs.

\section{EAD: Encoded Archival Description}

EAD is an archival description metadata standard that reflects and emphasizes the hierarchical nature of $\operatorname{ISAD}(G)$ 15. EAD fully enables the expression of multiple description levels central to most archive description 8 and reflects hierarchy levels present in the resources being described. EAD cannot be considered a one-to-one $\operatorname{ISAD}(G)$ implementation, although it does respect $\operatorname{ISAD}(G)$ principles and is useful for representing archival hierarchical structure. EAD is composed of three high-level components: <eadheader>, <frontmatter>, and <archdesc>.

The <eadheader > contains metadata about the archive descriptions and includes information about them such as title, author, and date of creation. The <frontmatter > supplies publishing information and is an optional element, while the <archdesc $>$ contains the archival description itself and constitutes the core of EAD. The <archdesc> may include many high-level sub-elements, most of which are repeatable. The most important element is the $\langle\mathrm{did}\rangle$ or descriptive identification which describes the collection as a whole. The $\langle$ did $\rangle$ element is composed

\footnotetext{
8 The concept of archive description is often referred the term finding aid defined in [14] as: "A description of records that gives the repository physical and intellectual control over the materials and that assists users to gain access to and understand the materials".
} 
of numerous sub-elements that are intended for brief, clearly designated statements of information and they are available at every level of description. Finally, the <archdesc> contains an element that facilitates a detailed analysis of the components of a fonds, the $\langle\mathrm{dsc}\rangle$ or description subordinate components. The $<\mathrm{dsc}>$ contains a repeatable recursive element, called $<\mathrm{c}>$ or component. A component may be an easily recognizable archival entity such as series, subseries or items. Components not only are nested under the <archdesc $>$ element, they usually are nested inside one another. Components usually are indicated with $<$ cN $>$ tag, where $N \in\{01,02, \ldots, 12\}$.

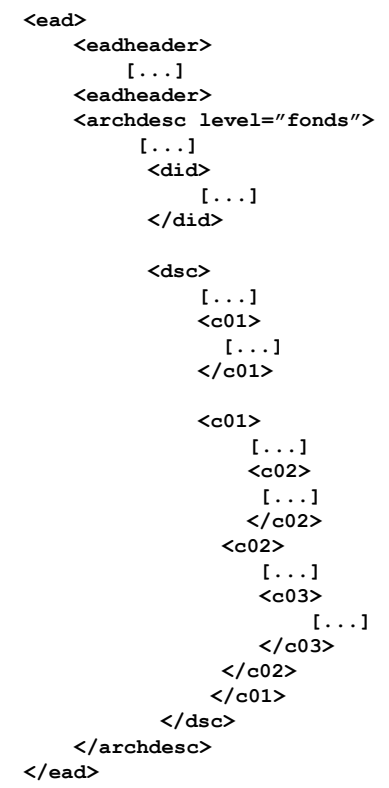

Fig. 1. Example of the structure of an EAD file

EAD reflects the archival structure and holds relations between entities in an archive. In addition, EAD encourages archivists to use collective and multilevel description, and because of its flexible structure and broad applicability, it has been embraced by many repositories $[10$.

On the other hand, EAD allows for several degrees of freedom in tagging practice, which may turn out to be problematic in the automatic processing of EAD files, since it is difficult to know in advance how an institution will use the hierarchical elements. The EAD permissive data model may undermine the very interoperability it is intended to foster. Indeed, it has been underlined that only EAD files meeting stringent best practice guidelines are shareable and searchable [19. Moreover, there is also a second relevant problem related to the level of material that is being described. Unfortunately, the EAD schema rarely requires a standardized description of the level of the materials being 
described, since the <level $>$ attribute is required only in the <archdesc $>$ tag, while it is optional in $\langle\mathrm{CN}\rangle$ components and in very few EAD files this possibility is used, as pointed out by [16. As a consequence, the level of description of the lower components in the hierarchy needs to be inferred by navigating the upper components, maybe up to the <archdesc>, where the presence of the $<$ level $>$ attribute is mandatory. Therefore, the access to individual items might be difficult without taking into consideration the whole hierarchy.

We highlight this fact in Figure 1 where we present the structure of an EAD file. In this example we can see the top-level components <eadheader> and $\langle$ archdesc $\rangle$ and the hierarchical part represented by the $\langle\mathrm{dsc}\rangle$ component; the $\langle$ level> attribute is specified only in the <archdesc $>$ component. Therefore, the archival levels described by the components of the $\langle$ dsc $\rangle$ can be inferred only by navigating the whole hierarchy.

Moreover, sharing and searching archival description might be made difficult by the typical size of EAD files which could be several megabytes with a very deep hierarchical structure. Indeed, each EAD file is a hierarchical description of a whole collection of items rather than the description of an individual item 20]. On the other hand, users are often interested in the information described at the item level, which is typically buried very deeply in the hierarchy and might be difficult to reach. Therefore, with respect to the three requirements, discussed in the previous section, EAD complies with both the context and the hierarchy requirements but it disregards the variable granularity one, since it might be problematic to access specific items without considering the whole data set.

\section{OAI-PMH and Dublin Core}

OAI-PMH is based on the distinction between two main components that are Data Provider and Service Provider. Data Providers are repositories that export records in response to requests from a software service called harvester. On the other hand, Service Providers are those services that harvest records form Data Providers and provide services built on top of aggregated harvest metadata.

The protocol defines two kinds of harvesting procedures: incremental and selective harvesting. Incremental harvesting permits users to query a Data Provider and ask it to return just the new, changed or deleted records from a certain date or between two dates. Selective harvesting is based on the concept of OAI set, which enables logical data partitioning by defining groups of records. Selective harvesting is the procedure that permits the harvesting only of metadata owned by a specified OAI set. [22] states that in OAI-PMH a set is defined by three components: setSpec which is mandatory and a unique identifier for the set within the repository, setName which is a mandatory short human-readable string naming the set, and setDesc which may hold community-specific XML-encoded data about the set.

OAI set organization may be flat or hierarchical, where hierarchy is expressed in setSpec field by the use of a colon [:] separated list indicating the path from the root of the set hierarchy to the respective node. For example if we define 


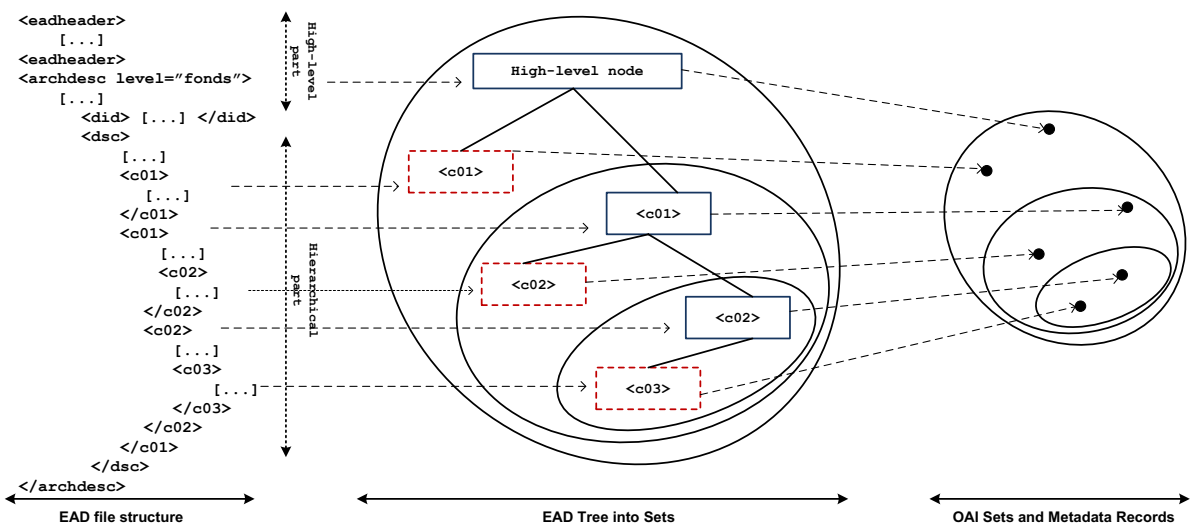

Fig. 2. Mapping EAD metadata into OAI Sets and DC metadata records

an OAI set for whose setSpec is " $A$ ", its sub-set " $B$ " would have " $A: B$ " as setSpec. In this case " $\mathrm{B}$ " is a proper sub-set of "A" $B \subset A$. When a repository defines a set organization it must include set membership information in the headers of items returned to the harvester requests. Harvesting from a set which has sub-sets will cause the repository to return metadata in the specified set and recursively to return metadata from all the sub-sets. In our example, if we harvest set A, we also obtain the items in sub-set B [21.

The Dublin Core (DC) metadata format is tiny, easy-to-move, shareable and remarkably suitable for a distributed environment. Thanks to these characteristics it is required as the lowest common denominator in OAI-PMH. Thus, DC metadata are very useful in information sharing but are not broadly used by archivists. Indeed, the use of DC seems to flatten out archive structure and lose context and hierarchy information. For this reason, even though DC is used in several contexts ranging from Web to digital libraries, it is less used in the archival domain. Nevertheless, we can apply it to the archival domain and meet the three requirements discussed above, if we use it in combination with OAI$\mathrm{PMH}$ : in this way, the OAI set provides us with context and hierarchy requirements compliance, while the DC metadata format gives us the expected variable granularity support.

\section{Expressing Archival Description Metadata by Using OAI-PMH and Dublin Core}

The proposed methodology permits the sharing of archival metadata in a distributed environment meeting archival needs. Our approach addresses context, hierarchy and variable granularity requisites exploiting DC and OAI-PMH peculiarities. We can express archive hierarchy and preserve meaningful relations between archival entities by levering the role of OAI sets. 
In Figure 2 we take up the EAD file structure presented in Figure 1 showing how its tree representation can be mapped in a collection of sets. More precisely, the union of the <eadheader> and the <archdesc> EAD high elements constitute the root of the EAD tree and the hierarchical part of EAD composed of descriptive subordinate components constitutes the remaining tree structure represented by the children's root. The main idea is to map the EAD tree into a combination of OAI sets and DC metadata records, which maintain the context and hierarchy expressed by EAD and the variable granularity guaranteed by DC.

To present our methodology it is worthwhile defining two fundamental characteristics of tree data structure: internal and external nodes. An internal node is defined to be a node having at least one child, instead an external node is defined as a node without children 11. In Figure 2 we can see three external nodes drawn with a sketched shape and three internal nodes of which one is the tree root.

We propose a methodology to map the structure of EAD files into several DC metadata records and OAI sets. As far as the mapping of the actual content of EAD items into DC records is concerned, we adopt the mapping proposed by Prom and Habing [18. We differ from [18] in the way in which the structure of EAD files is translated into OAI sets and DC records, as we will discuss in more detail at the end of the section.

Our methodology, shown in Figure 2, is constituted by three main steps:

1. OAI sets: creation of an OAI set for each internal node of the tree.

2. Metadata set record: a metadata record for each set constituted in the step one must be created; we call these records metadata set records because they are built contextually with the OAI set.

3. Metadata record: a metadata record for each external node of the tree must be created.

Step one establishes that for each internal node of the EAD tree an OAI set is created. In order to define an OAI set the setSpec parameter must be settled with a unique value as we shown in Figure 3 , This procedure is repeated recursively for each internal node in every level of the hierarchy. In the example we proposed in Figure 2 a sub-set is created from the root's internal node child labeled with c01 and recursively, a sub-set of this sub-set is created from its internal node child labeled with c02.

The second step is based on the creation of what we called a metadata set record for each set defined in the first step. Indeed, internal nodes maintain what in a very general way could be defined collection-level information; this information must be retained in the OAI environment. The OAI-PMH protocol considers metadata records basic and privileged information units, thus collection-level information must be kept in a metadata record. These records are strictly linked with sets created by the EAD tree internal nodes and for this reason they are called metadata set records. A metadata set record has the structure of a normal DC metadata record but it retains a different granularity of information. Metadata set records enable incremental harvesting. Indeed when a set changes 
its composition, the date field is updated in the metadata set record enabling a Service Provider to harvest the updated information. According to OAI sets functioning, when a Service Provider requires the records belonging to a set, it can harvest also all the records belonging to its sub-sets, so that all data hierarchy can be harvested.

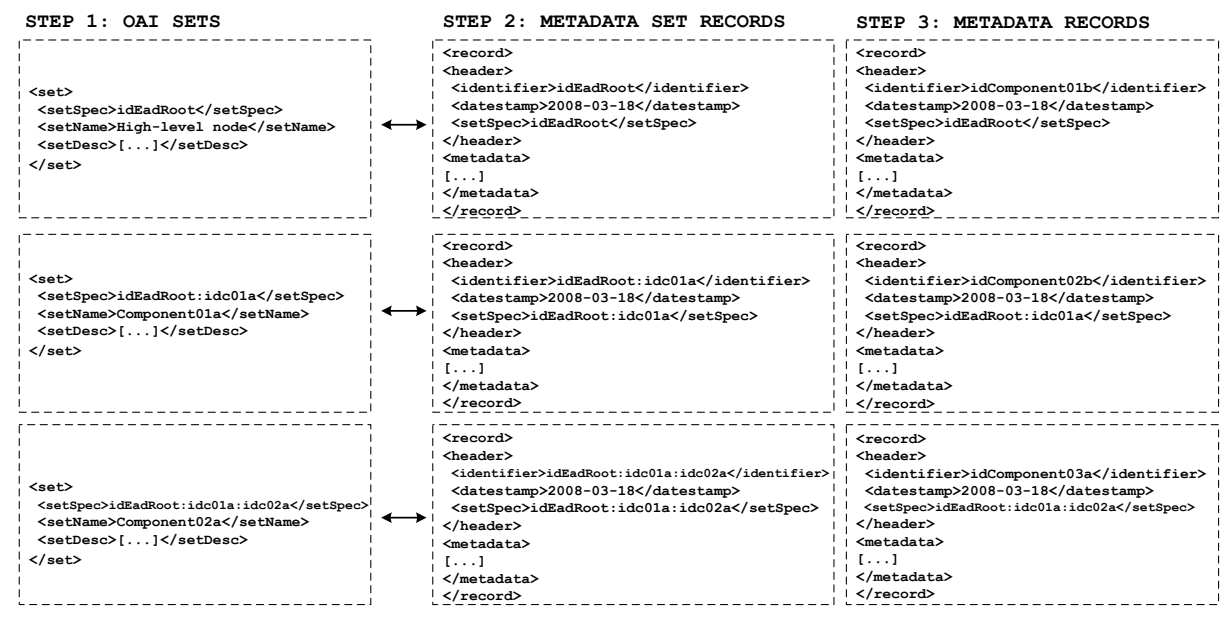

Fig. 3. OAI sets and metadata records generated by the proposed methodology

We impose the identifier of set metadata record to be the same as the OAI set's SetSpec value, so that the relations between these two entities is straightforward and an implicit link between these two entities is created. Within these two steps we retain context information expressing and preserving archive hierarchy through the specified OAI sets organization. Metadata set records permit access to different resource levels meeting variable granularity metadata requisite.

In the third step a metadata record is created for each external node of the EAD tree. This metadata record belongs to the set created from the external node's father in step one; the header of the metadata record must contain set membership information as specificated by the OAI-PMH protocol.

In Figure 3 are shown the OAI sets and metadata records resulting by the application of the proposed methodology to the EAD file presented in Figure 1. After the application of the first step an OAI set is created from the toplevel components <eadheader > and <archdesc> composing the root of the EAD tree showed in Figure 2 The setSpec value of this OAI set is: "idEadRoot". Recursively are build the OAI sets from the two internal nodes of the EAD tree that are $\langle\mathrm{c01}\rangle$ and $\langle\mathrm{c02}\rangle$. The $\langle$ setSpec $\rangle$ value of the OAI set build from <c01> is: "idEadRoot:idc01a"; instead <setSpec> value of the OAI set build from <c02> is: "idEadRoot:idc01a:idc02a".

The second step generated a metadata set record for each OAI set created in the first step. Indeed, as we can see in Figure 3 there are three OAI sets and 
three metadata set records. Each metadata set record has the identifier equals to the setSpec value of the related OAI set; the first metadata record identifier is "idEadRoot", the second one is "idEadRoot:idc01a" and the third one is "idEadRoot:idc01a:idc02a". Furthermore, each of these three set metadata records must belong to its respective OAI set; indeed the setSpec value is reported in the record header as specified by the OAI-PMH protocol.

The third step created a metadata record for each EAD tree external node;

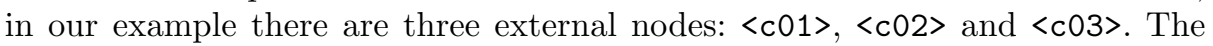
three metadata records created from these nodes must retain set membership information in their headers. Indeed as we can see in Figure 3 the first record belongs to the OAI set with "idEadRoot" setSpec value, the second one to the OAI set with "idEadRoot:idc01a" setSpec value and the third one to the OAI Set with "idEadRoot:idc01a:idc02a" setSpec value. At the end of these three steps the considered EAD file is mapped into three OAI sets and six metadata records.

The proposed solution addresses the shortcomings of EAD when it has to be used in a distributed environment and with variable granularity access to the resources. Indeed, EAD items are mapped into different DC metadata records which are shareable metadata, and natively supported by OAI-PMH. Furthermore, context and hierarchy are preserved and expressed in a straightforward manner exploiting the native functionalities of OAI-PMH and DC metadata format. Indeed, the organization into OAI sets reflects archive hierarchy and each metadata record also maintains in its header the membership information which is essential to go up again to related entities and to express contextual information.

This methodology could be compared with the Prom-Habing approach [18, where each EAD file is divided into several DC metadata records and also a mapping between EAD values and DC terms is proposed. In this approach every DC metadata record generated from an EAD file contains an XPointer 9 reference pointing back to the original node in the EAD file; this is the identifier of the DC metadata record. XPointer are also used to link every DC metadata record to the part of the source EAD file where the original reference is found. With this approach hierarchy is preserved thanks to the many links to the original file; therefore there is a strong dependency between OAI records and the EAD file. Indeed, archive hierarchy is preserved by means of the original EAD file and it has been underlined [17] that the searcher will need to rely on a link to the full archive description in order to view the all important context; thus a potential user must follow a link and consult the original EAD file to have context information. In contrast, our approach permits to keep archival metadata independent of the original EAD file, without loosing any context information.

Furthermore, our approach can be applied also independently of the EAD standard; indeed, we can also create archival description metadata from scratch by exploiting OAI set and DC records, as explained above; these archival description metadata will accomplish all archive needs and requirements, discussed

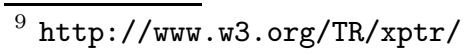


in Section 2, and will be easily shared through OAI-PMH. From this point of view, the proposed lossless mapping between EAD and our methodology can be considered as a proof of the validity of our approach for describing archival resources on their own.

\section{Conclusions}

We have discussed the use of archival resources in a digital and possibly distributed environment. Firstly, we defined the requisites which must be satisfied in order to obtain shareable metadata and to retain all the fundamental characteristics of archival resources. Then, we presented a methodology for creating sharable archival description metadata which exploit the synergy between the OAI-PMH protocol and the DC metadata format. We have also shown the validity of our approach by demonstrating that EAD metadata can be mapped into our methodology without losing information. The methodology can be applied backwards generating a new EAD file from the obtained DC metadata; this new generated EAD file might have a slightly different structure compared to the original one, but it brings exactly the same informational content.

\section{Acknowledgements}

The work reported has been supported by a grant from the Italian Veneto Region. The study is also partially supported by the TELplus Targeted Project for digital libraries, as part of the eContentplus Program of the European Commission (Contract ECP-2006-DILI- 510003).

\section{References}

1. Agosti, M., Ferro, N., Silvello, G.: An Architecture for Sharing Metadata among Geographically Distributed Archives. In: Thanos, C., Borri, F., Candela, L. (eds.) Digital Libraries: Research and Development. LNCS, vol. 4877, pp. 56-65. Springer, Heidelberg (2007)

2. Agosti, M., Ferro, N., Silvello, G.: Proposta metodologica e architetturale per la gestione distribuita e condivisa di collezioni di documenti digitali. Archivi. 2(2), 49-73 (2007)

3. Candela, L., Castelli, D., Ferro, N., Koutrika, G., Meghini, C., Pagano, P., Ross, S., Soergel, D., Agosti, M., Dobreva, M., Katifori, V., Schuldt, H.: The DELOS Digital Library Reference Model. Foundations for Digital Libraries. ISTI-CNR at Gruppo ALI, Pisa, Italy (November 2007)

4. Candela, L., Castelli, D., Manghi, P., Pagano, P.: OAI-Aggregator Services for Enhanced Cross-Repository Services. In: Post-proceedings of the Third Italian Research Conference on Digital Library Systems (IRCDL 2007). ISTI-CNR at Gruppo ALI, Pisa, Italy, pp. 61-66 (November 2007)

5. Cook, T.: The Concept of Archival Fonds and the Post-Custodial Era: Theory, Problems and Solutions. Archiviaria 35, 24-37 (1993) 
6. Duranti, L.: Diplomatics: New Uses for an Old Science. Society of American Archivists and Association of Canadian Archivists in association with Scarecrow Press (1998)

7. Gilliland-Swetland, A.J.: Enduring Paradigm, New Opportunities: The Value of the Archival Perspective in the Digital Environment. Council on Library and Information Resources (2000)

8. Gradmann, S.: Interoperability of Digital Libraries: Report on the work of the EC working group on DL interoperability. In: Seminar on Disclosure and Preservation: Fostering European Culture in The Digital Landscape. Lisbon, Portugal (September 2007)

9. International Council on Archives. $\operatorname{ISAD}(\mathrm{G})$ : General International Standard Archival Description, 2nd edn. International Council on Archives, Ottawa (1999)

10. Kiesling, K.: Metadata, Metadata, Everywhere - But Where Is the Hook? OCLC Systems \& Services 17(2), 84-88 (2001)

11. Knuth, D.E.: The Art of Computer Programming, 3rd edn., vol. 1. Addison-Wesley, Reading (1997)

12. MacNeil, H., Wei, C., Duranti, L., Gilliland-Swetland, A., Guercio, M., Hackett, Y., Hamidzadeh, B., Iacovino, L., Lee, B., McKemmish, S., Roeder, J., Ross, S., Wan, W., Zhon Xiu, Z.: Authenticity Task Force Report. InterPARES Project, Vancouver, Canada (2001)

13. Society of American Archivists. Encoded Archival Description: Tag Library, ver. 2002. Society of American Archivists (2003)

14. Pearce-Moses, R.: Glossary of Archival And Records Terminology. Society of American Archivists (2005)

15. Pitti, D.V.: Encoded Archival Description. An Introduction and Overview. D-Lib Magazine 5(11) (1999)

16. Prom, C.J.: Does EAD Play Well with Other Metadata Standards? Searching and Retrieving EAD Using the OAI Protocols. Journal of Archival Organization 1(3), 51-72 (2002)

17. Prom, C.J.: Reengineering Archival Access Through the OAI Protocols. Library Hi Tech. 21(2), 199-209 (2003)

18. Prom, C.J., Habing, T.G.: Using the Open Archives Initiative Protocols with EAD. In: Proc. 2nd ACM/IEEE Joint Conference on Digital Libraries (JCDL 2002), pp. 171-180. ACM Press, New York (2002)

19. Prom, C.J., Rishel, C.A., Schwartz, S.W., Fox, K.J.: A Unified Platform for Archival Description and Access. In: Proc. 7th ACM/IEEE Joint Conference on Digital Libraries (JCDL 2007), pp. 157-166. ACM Press, New York (2007)

20. Shreeves, S.L., Kaczmarek, J.S., Cole, T.W.: Harvesting Cultural Heritage Metadata Using the OAI Protocol. Library Hi Tech. 21(2), 159-169 (2003)

21. Van de Sompel, H., Lagoze, C., Nelson, M., Warner, S.: Implementation Guidelines for the Open Archive Initiative Protocol for Metadata Harvesting - Guidelines for Harvester Implementers. Technical report, Open Archive Initiative, p. 6 (2002)

22. Van de Sompel, H., Lagoze, C., Nelson, M., Warner, S.: The Open Archives Initiative Protocol for Metadata Harvesting (2nd edn.). Technical report, Open Archive Initiative, p. 24 (2003) 